\title{
Aprendizagem e Procrastinação: Uma Revisão de Publicações no Período 2005-2015
}

\section{Learning and Procrastination: A Review of Publications from 2005 to 2015}

\author{
Rony Rodrigues Correia * \\ Pedro Jácome de Moura Júnior \\ Universidade Federal da Paraíba
}

\begin{abstract}
Aprendizagem e procrastinação são dois fenômenos inter-relacionados. Além disso, tanto aprendizagem quanto procrastinação parecem impactar todas as esferas da vida social moderna. O objetivo deste artigo é identificar evidências científicas do período 2005 a 2015 sobre as relações conceituais entre os dois fenômenos. Por meio de revisão sistemática da literatura é possível identificar três categorias de conceitos centrais envolvendo esses dois construtos (autoeficácia, autorregulação e o papel das emoções e dos valores do indivíduo), além do uso de ferramentas para redução da procrastinação no ambiente da aprendizagem. $\mathrm{O}$ artigo apresenta uma síntese das proposições elaboradas nos estudos prévios levantados sobre as relações dessas categorias com a aprendizagem e a procrastinação. Para auxiliar na apreensão das proposições apresentadas, é elaborado um mapa conceitual das relações entre os conceitos investigados pela revisão. Conclui-se que há viés epistemológico nas visões sobre os dois fenômenos, sugerindo a oportunidade de exploração do tema por meio de novas abordagens.
\end{abstract}

Palavras-chaves: Aprendizagem, Revisão, Eficácia, Reguladora, Autonomia educativa.

Learning and procrastination are two interrelated phenomena. In addition, both learning and procrastination appear to impact all domains of modern social life. The paper aims at identifying scientific evidences of publications between the years of 2005 and 2015 on the conceptual relationship between both phenomena. The systematic literature review method presents three categories of core concepts involving those two constructs (self-efficacy, self-regulation and the role of individual emotions and values), and the use of tools to reduce procrastination in learning environments. The paper presents an overview of the statements presented on the previous studies about the relationship of these categories with learning and procrastination. In order to help the apprehension of the presented statements, it is developed a conceptual map of the relationships between the concepts investigated by the review. We conclude that there is a bias in the epistemological views on the two phenomena, suggesting an opportunity to explore new approaches.

Keywords: Learning, Review, Effectiveness, Regulatory, Educational autonomy.

*Contacto: ronyre@gmail.com

ISSN: $1696-4713$

www.rinace.net/reice/

revistas.uam.es/reice
Recibido: $\quad 15$ de julio 2016

$1^{\text {a }}$ Evaluación: 28 de octubre 2016

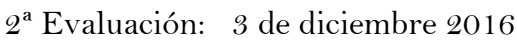

Aceptado: $\quad 3$ de enero 2017 


\section{Introdução}

A aprendizagem é um processo integrado em praticamente todas as esferas da vida social moderna. Diversas são as abordagens e perspectivas que tentam compreender o fenômeno da aprendizagem. Numa delas, a aprendizagem em ação (action learning, no original em inglês), o ser humano aprende por meio da interação ao se engajar na busca de soluções para problemas da vida real. Nessa abordagem, aprender é se envolver em tarefas e desafios (Raelin, 2009).

Entretanto, alguns indivíduos postergam ao máximo seu envolvimento em tarefas para alcançar algum objetivo. A procrastinação é um comportamento social complexo e múltiplo que envolve diversas dimensões, apresentando-se de forma diferenciada nos domínios social, de saúde, trabalho ou criativo. Se por um lado, o comportamento procrastinador pode ser encarado como benéfico ao reduzir os níveis de absenteísmo e turnover em tarefas entediantes (Harris e Sutton, 1983), por outro, pode ser percebido como maléfico ao produzir mais estresse e doenças após a finalização da tarefa adiada (Tice e Baumeister, 1997).

Mesmo estando relacionado a traços de personalidade (Watson, 2001), o comportamento procrastinador deve ser entendido como um traço multidimensional, impactando diversos aspectos da vida íntima e também social do indivíduo. Dentre esses aspectos, a aprendizagem parece receber especial atenção. Por exemplo, características de insucesso de autorregulação presentes na procrastinação, como baixa motivação para a conquista de objetivos ou a desorganização, evocam abordagens fundamentais em modelos de aprendizagem, como a aprendizagem autorregulada (Howell e Watson, 2007).

Assim, torna-se desejável uma investigação sobre as relações entre procrastinação e aprendizagem, uma vez que existem conceitos centrais e relacionados envolvendo os dois fenômenos. Para abordar o tema, este estudo propõe uma revisão sistemática da literatura (Sampaio e Mancini, 2007) com o intuito de responder à seguinte questão de pesquisa: quais as evidências científicas dos últimos dez anos sobre as relações conceituais entre procrastinação e aprendizagem? O foco da investigação envolveu o período entre os anos de 2005 e 2015 com o intuito de propor as relações mais atuais entre os dois conceitos e distinguir linhas de pesquisas recentemente adotadas, uma vez que aprendizagem (Merriam e Bierema, 2014) e procrastinação (Watson, 2001) seguem, cada um, cursos de investigação divergentes. Além disso, desde o trabalho de Van Eerde (2003) não se identificou compilação relevante de publicações sobre o tema.

Não foi prevista nenhuma abordagem específica para análise dos dois fenômenos, ou seja, qualquer perspectiva de aprendizagem ou definição de procrastinação foram consideradas para o levantamento realizado. O pressuposto do estudo é que todo fenômeno é reduzido em termos teóricos para viabilização da pesquisa. Por exemplo, encarar a aprendizagem apenas como uma mudança de sentido na existência do ser humano deixa de considerar aspectos importantes relacionados a processos cerebrais envolvidos na aprendizagem. Da mesma forma, procrastinar não é só deixar para amanhã o que se pode fazer hoje, uma vez que o fenômeno possui dimensões cognitivas, afetivas e comportamentais.

$\mathrm{Na}$ próxima seção, serão apresentadas as principais abordagens teóricas de aprendizagem e uma revisão dos conceitos de procrastinação para compor um referencial teórico. Embora algumas revisões sistemáticas da literatura já apresentem passos 
metodológicos após essa seção inicial de introdução ou contextualização (p. ex. De Moura e Bellini, 2013), optou-se nesse estudo pela elaboração de referencial teórico que permita discussão aprofundada, a posteriori, dos resultados encontrados. Em seguida, ao referencial teórico será apresentado o protocolo de pesquisa que compõe a metodologia adotada. As seções de resultados e discussão apresentam epítome das evidências encontradas e categorias de conceitos relacionados. Por fim, são apresentadas as conclusões do trabalho.

\section{Referencial teórico}

\subsection{Perspectivas teóricas da aprendizagem}

A aprendizagem pode ser conceituada de diversas maneiras. Conceitos mais atuais de aprendizagem envolvem obter mais conhecimento, memorizar e reproduzir, aplicar fatos ou procedimentos, entender, ver algo de uma forma diferente ou transformar-se como pessoa. Essas formas divergentes podem ser conduzidas por diferentes pessoas ou pela mesma pessoa em circunstâncias diversas, ou ainda para propósitos diferenciados (Carnell e Lodge, 2001).

Mais recentemente, as perspectivas teóricas da aprendizagem tentam explicar como a aprendizagem ocorre e como essa explicação pode ser referenciada na prática. Merriam e Bierema (2014) selecionam cinco orientações que visam explicar a aprendizagem na perspectiva de sua aplicação.

A explicação "behaviorista" acredita que o comportamento humano é resultado do arranjo de estímulos particulares no ambiente. Assim, a aprendizagem nessa perspectiva é definida como uma mudança observável em um comportamento. A ênfase da explicação behaviorista está na recompensa esperada pelo indivíduo em empreender atividades de aprendizagem, nos estímulos que os aprendizes recebem do ambiente e, por fim, na observação sistemática do comportamento. A despeito de várias pesquisas empíricas utilizarem essa visão para implantar estratégias de aprendizagem, ela é descrita como mecanicista e controladora.

A explicação "humanista" considera os seres humanos como detentores de potencial para crescimento e desenvolvimento. Ela acredita que as pessoas são livres para realizar escolhas e, com isso, determinar seu comportamento. Dessa forma, o ser humano possui aspirações para tornar-se realizado, sendo essa sua motivação para aprender. Tal abordagem de aprendizagem está centrada no aprendiz, influenciada pela psicologia humanista de Carl Rogers (1902-1987). O aprendiz dá significado à aprendizagem, imprimindo uma qualidade de envolvimento pessoal no processo. As três principais teorias da aprendizagem de adultos -andragogia, aprendizagem autodirecionada e aprendizagem transformativa- têm fundamentos na explicação humanista.

A explicação "cognitivista" considera aprendizagem um processo mental, onde ocorrem insights (o momento quando a solução de um problema torna-se clara), processamento de informação, resolução de problemas, acesso à memória e processos cerebrais. A explicação "cognitivista" foca como o cérebro é utilizado na aprendizagem e como nossos sentidos são ativados para processar informação. Tal aprendizagem é encarada como uma ferramenta para o desenvolvimento cognitivo e o incremento da memória do indivíduo. 
A explicação "cognitivista social" vê a aprendizagem como um processo social e restrito pelo contexto no qual o indivíduo está posicionado. A aprendizagem, nessa visão, consiste na observação dos outros e como os comportamentos observados são modelados pelo indivíduo. Há três pilares interativos e recíprocos para o processo: a própria aprendizagem, o indivíduo e o ambiente que media os dois primeiros.

Por fim, a explicação "construtivista" foca como as pessoas criam sentido a partir da experiência, ou seja, a aprendizagem é a construção do significado da experiência. O processo consiste em construir e trocar significados pessoais e relevantes da experiência da aprendizagem. Essa explicação pode posicionar a aprendizagem num tipo de cognição situada socioculturalmente, onde os significados são valorados em determinado ambiente. Nesse caso, indivíduos compartilham e aprendem uns dos outros.

\subsection{Procrastinação}

A procrastinação consiste em uma tendência a postergar aquilo que é necessário para alcançar algum objetivo (Lay, 1986). Ela não é um fenômeno linear, geralmente não podendo ser medido como expressão de um único comportamento. Dessa maneira, um indivíduo deixa de cumprir tarefas não apenas por efeitos deliberativos, mas também porque não sabe como executar a tarefa ou possui aversão à mesma.

Diversos estudos associam a procrastinação com aspectos negativos ou maléficos. Pessoas com baixa competência acadêmica percebida possuem uma tendência a procrastinar, sendo causada pelo medo de falhar na tarefa (Haghbin, McCaffrey e Pychyl, 2012). A procrastinação tanto é associada ao medo de falhar como ao medo de vencer, ou ainda como uma forma de rebelião à autoridade, em que é apresentada uma desorganização neurótica em nível cognitivo e em atividades rotineiras (Lay, 1986). Apesar de não serem evidentes as relações entre procrastinação e inteligência, indivíduos procrastinadores produzem trabalhos inferiores, relatam mais estresse e doenças após a finalização de tarefas e são pior avaliados no desempenho dessas tarefas, resultando num estado de desânimo (Tice e Baumeister, 1997).

Ocorre que, a despeito das pressuposições perniciosas sobre procrastinadores, uma parte deles possuem características atitudinais e comportamentais desejáveis, ainda que possam expressar o mesmo nível de procrastinação daqueles que não possuem tais características. Essa parcela de procrastinadores prefere trabalhar sobre pressão e suas decisões sobre procrastinação são deliberadas. Eles são chamados de "procrastinadores ativos" e compartilham mais características e ideias com indivíduos que usualmente não procrastinam do que com os "procrastinadores passivos", já que tendem a apresentar altos níveis de uso efetivo do tempo, gerenciam seu tempo e apresentam autoeficácia (Chu e Choi, 2005). A autoeficácia é uma conviç̧ão que um indivíduo possui de sua capacidade em executar de maneira bem-sucedida um comportamento requerido a fim de produzir resultados (Bandura, 1977).

Portanto, nem todo comportamento procrastinador é nocivo. Por exemplo, no âmbito da procrastinação acadêmica, a alta identificação do indivíduo com a tarefa e a baixa regulação externa aumentam a procrastinação ativa. Por sua vez, a alta regulação externa e a baixa motivação intrínseca aumentam a procrastinação passiva. Ao menos em atividades acadêmicas, a procrastinação ativa está associada com formas autônomas de motivação, levando a estados mais elevados de autodeterminação (Seo, 2013). 
Por fim, podem ser elaboradas estratégias ou tratamentos para conter o comportamento procrastinatório e seus efeitos. Programas de terapia em grupo mostraram-se eficazes ao reduzir comportamento procrastinatório em estudantes. O tratamento foi efetivo nos casos onde a procrastinação estava associada com sentimentos de desvalorização e autocrítica (Ozer, Demir e Ferrari, 2013).

\section{Metodologia}

Adotou-se revisão sistemática da literatura (RSL) para obtenção de resposta à questão de pesquisa, já que esse método provê ferramenta adequada para síntese dos estudos científicos de melhor qualidade sobre um determinado tópico ou questão de pesquisa. Comparada com uma revisão utilizando seleção de fontes ad hoc, a RSL é um processo metodologicamente rigoroso para revisão de resultados de pesquisas (Kitchenham et al., 2009).

Este estudo empregou a abordagem de Sampaio e Mancini (2007) para desenvolvimento da RSL. Para eles, uma revisão sistemática da literatura deve acompanhar um protocolo, seguindo alguns passos. O Passo 1 envolve a preparação de uma questão de pesquisa bem formulada. Os conceitos principais sobre os fenômenos procrastinação e aprendizagem permitiram a elaboração da questão de pesquisa. Em seguida, o Passo 2 envolve a busca de evidências. Tal busca é iniciada com a definição de termos ou palavras-chave que norteiam a pesquisa. Para esse estudo foram utilizados os termos "procrastination" e "learning". Foram utilizados os termos em inglês por se tratar da língua na qual mais se publica evidências científicas. Além disso, diversas outras línguas elaboram os metadados dos seus artigos, como resumos e palavras-chave, também em inglês.

Ainda no Passo 2 é necessário definir as fontes primárias (bases de dados, bibliotecas, indexadores etc.) dos estudos primários (artigos científicos, nesse caso) que serão pesquisados. Para esse estudo, foi definida a base de dados do Portal de Periódicos da CAPES. Além de oferecer os artigos completos para leitura (não apenas as referências ou resumos), a base da CAPES permite realizar pesquisas customizadas em campos personalizados dos artigos. Essa ferramenta do portal de periódicos em questão foi útil para a viabilização desse estudo, já que os termos selecionados “procrastination” e “learning” são muito genéricos. Dessa forma, foi possível eliminar estudos que apenas citavam os termos e não faziam parte do interesse de pesquisa. Foram pesquisados os termos no Portal de Periódicos CAPES no campo “Assunto” entre os períodos 2005 e 2015, retornando estudos que, de fato, tratavam de aprendizagem e procrastinação. Os campos preenchidos no portal estão exibidos na figura 1.

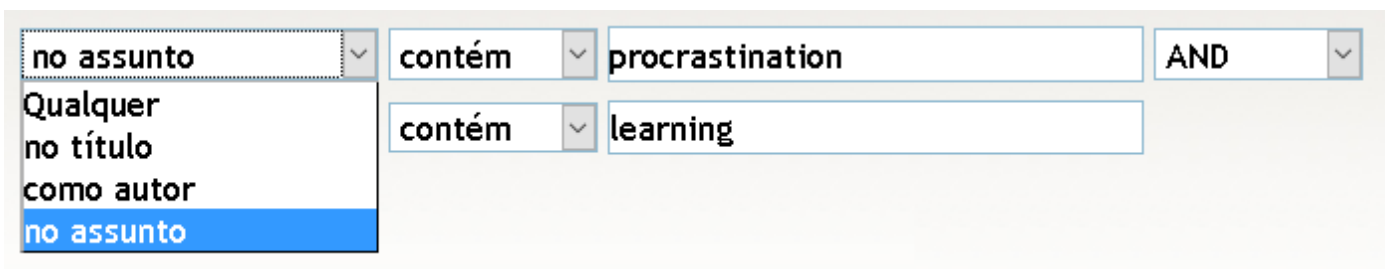

Figura 1. Pesquisa avançada do portal de periódicos CAPES Fonte: Portal de Periódicos (CAPES, 2016). 
O Passo 3 envolve a revisão e seleção dos estudos. Após selecionar os artigos retornados pela base científica, o pesquisador deve revisar e selecionar os estudos que apropriadamente dão suporte à questão de pesquisa. Para esse estudo, foram verificados os títulos, resumos e palavras-chave como critério de inclusão ou exclusão nesse passo. O Passo 4 realiza uma análise da qualidade metodológica dos estudos incluídos e sua descrição. Cabe aqui uma ressalva para este passo pois como foram incluídos artigos que possuíam métodos científicos diversos, não foram considerados aspectos epistemológicos como critério de qualidade, mas, sim, sua adequação para a resposta da questão de pesquisa do artigo revisado. Por fim, o Passo 5 envolve a apresentação dos resultados.

\section{Resultados e discussão}

A aplicação do protocolo de pesquisa, conforme descrito, resultou na seleção de 41 artigos. A leitura de títulos, resumos e palavras-chave dos artigos retornados causou a exclusão de 11 estudos (Blair, 2008; Bruhm, 2008; Case, 2009; Finigan, 2008; Hederih e Čagran, 2014; Kim, 2012; O’Driscoll, 2008; Panadero e Alonso-Tapia, 2014; Pychyl, 2008; Sugiyama, Hirowatari, Tsuiki e Yamamoto, 2013; Wright, 2008) por não tratarem especificamente do interesse da pesquisa, não serem possíveis de tradução ou não apresentarem evidência científica primária. Portanto, após a exclusão, a RSL foi conduzida com 30 artigos em 25 periódicos distintos.

Dentre os estudos selecionados, 26 artigos (aproximadamente 87\%) seguem a abordagem quantitativa com aplicação de questionários e análises estatísticas, ou o desenvolvimento de experimentos. Um artigo (Alias, 2012) utilizou a abordagem de design/development research, empregando intervenção centrada nos participantes com orientação à ação e à teoria. A tabela 1 apresenta um levantamento das técnicas de análises dos dados empregadas nos artigos pesquisados.

Observa-se que a carência de abordagens qualitativas nos estudos das relações entre procrastinação e aprendizagem sugere tradição de pesquisa funcionalista na abordagem desses dois fenômenos. Tanto a procrastinação quanto a aprendizagem (Merriam e Bierema, 2014) envolvem complexidades que demandam novas relações para expandir o entendimento de sua manifestação. Uma mudança metodológica pode ajudar a explicar resultados aparentemente conflitantes nas pesquisas que abordam a temática.

Como exemplo de pesquisas conflitantes, observa-se que exaustão, stress, ansiedade e falta de interesse foram positivamente correlacionados com bloqueio na escrita acadêmica, procrastinação e perfeccionismo exacerbado (Lonka et al., 2014). Por outro lado, observa-se também que procrastinação não foi positivamente correlacionada com desempenho acadêmico associado ao sistema de notas e a satisfação de vida do estudante num ambiente de aprendizagem a distância (Klingsieck et al., 2012). Uma abordagem qualitativa, em que pode haver uma maior aproximação do pesquisador com o fenômeno pesquisado, poderia gerar novas relações entre procrastinação e bem-estar do indivíduo, além de aprofundar o processo de seleção de estratégias de aprendizagem. 
Tabela 1. Técnicas de análise dos dados empregadas nos artigos pesquisados

\section{TIPO DE ESTUdO OU}

ANÁLISE

\section{ESTUDOS}

Análise Fatorial, Análise de

Dietz, Hofer e Fries, 2007; Hen e Goroshit, 2012; Kennedy e Caminhos ou Modelagem de Equações Estruturais Tuckman, 2013; Lonka et al., 2014; Macher, Paechter, Papousek e Ruggerii, 2012; Michinov, Brunot, Le Bohec, Juhel e Delaval, 2011 ; Wolters e Benzon, 2013; You e Kang, 2014.

Artino e Stephens, 2009; Cao, 2012; Corkin, Yu e Lindt, 2011 ;

Análise de Regressão Howell e Watson, 2007; Katz, Eilot e Nevo, 2014; Kim e Seo, 2013; Lubbers, Van der Werf, Kuyper e Hendriks, 2010; Wolters e Hussain, 2015.

Bong, Hwang, Noh e Kim, 2014; González-Brignardello e Sánchez-

Outras Análises

Correlacionais e/ou Elvira-Paniagua, 2013; Klingsieck, Fries, Horz e Hofer, 2012; Peled e Sarid, 2010; Rodarte-Luna e Sherry, 2008; Romano, Wallace, Helmick, Carey e Adkins, 2005; Vahedi, Farrokhi, Gahramani e Issazadegan, 2012; Warnock, Bingham, Driscoll, Fromal e Rouse, 2012; Wäschle et al., 2014a; Zusho e Barnett, 2011.

Multivariadas

Akin, 2012; Sampaio-Maia, Maia, Leitão, Amaral e Vieira-Marques, 2013; Wäschle et al., 2014b.

Experimentos

Alias, 2012

Design/Development

Research

Fonte: Elaborado pelos autores.

\subsection{Aprendizagem e procrastinação: Que relações?}

Os artigos localizados nesta RSL tratavam das relações mais recentes entre procrastinação e aprendizagem. Nesta seção, serão apresentadas as categorias de conceitos adjacentes aos dois fenômenos e suas principais proposições.

\subsubsection{Autoeficácia}

O conceito de autoeficácia está intimamente associado com a visão cognitivista social da aprendizagem (Knowles, Holton e Swanson, 2011). Dessa maneira, a autoeficácia é um tipo de reforço utilizado para a aprendizagem ocorrer, indo além da observação e modelagem do comportamento alheio. Indivíduos com altos índices de autoeficácia tendem a estabelecer objetivos mais desafiadores, empenham-se de forma mais incisiva, persistem por mais tempo na tarefa, sentem-se melhor e conseguem melhores resultados (Yeo e Neal, 2013). Observa-se que indivíduos que adotam o objetivo desafiador de aprender tudo o que há para aprender em um curso não procrastinam, ao passo que indivíduos que se limitam apenas a evitar falhar no processo de aprendizagem tendem a procrastinar (Howell e Watson, 2007).

As relações entre procrastinação e autoeficácia num processo de aprendizagem determinam que esses dois conceitos são circulares. A procrastinação tende a se reforçar como um círculo vicioso. Já a autoeficácia resulta num círculo virtuoso que ajuda estudantes a superar a tendência a procrastinar. Estudantes continuam ou reforçam a procrastinação ao estabelecerem baixas metas de realização. Por outro lado, quanto maior autoeficácia for experimentada, menor a tendência a procrastinar (Wäschle et al., 2014a). 
A autoeficácia também pode ser considerada um processo de aprendizagem autorregulada adaptativa. Além disso, os processos de aprendizagem autorregulatória estão muito associados com procrastinação pois essa última é considerada também um comportamento de falha autorregulatória. A despeito da classificação clássica (Chu e Choi, 2005) entre procrastinadores ativos e passivos, comportamentos autorregulatórios efetivos e inefetivos podem ser classificados como "atraso ativo" e "procrastinação", respectivamente. Indivíduos que praticam atraso ativo diferem de procrastinadores nos seus níveis de autoeficácia, percepções de tempo e em resultados pessoais. Além disso, procrastinação está negativamente relacionada com processos autorregulatórios, como a autoeficácia, e atraso ativo está relacionado positivamente (Corkin, Yu e Lindt, 2011).

As relações entre procrastinação e autoeficácia podem ser moderadas e mediadas por outras variáveis. Uma delas -a motivação autônoma- consiste em um tipo de motivação intrínseca ao indivíduo, onde o locus de regulação da ação reside em identificar a importância do comportamento. Quanto maior o aspecto autônomo da motivação, maior a qualidade do envolvimento na tarefa, a experiência emocional e o sentimento de bemestar da pessoa (Katz, Eilot e Nevo, 2014).

Apesar de a baixa autoeficácia ser uma das razões centrais da procrastinação, os efeitos da motivação autônoma nas relações entre esses dois conceitos fazem com que a procrastinação não possa ser reduzida simplesmente para um direcionamento na autoeficácia do estudante. Ela deve ser suportada para adotar tipos mais autônomos de motivação. Assim, a autoeficácia influencia o tipo de motivação, que por sua vez, influencia o nível de procrastinação (Katz, Eilot e Nevo, 2014).

Às vezes duas facetas de um mesmo fenômeno da aprendizagem possuem relações diversas com a procrastinação. $\mathrm{O}$ perfeccionismo refere-se a um traço de personalidade da pessoa que estabelece metas dificultosas para si e possui avaliação crítica do desempenho em frente a esses objetivos. O perfeccionismo é considerado um construto multidimensional. Pode ser considerado "auto-orientado", quando os padrões de perfeccionismo são impostos pelo próprio indivíduo ou "socialmente prescritos" quando esses padrões são impostos por outros. O perfeccionismo auto-orientado é negativamente relacionado com a procrastinação acadêmica e o perfeccionismo socialmente prescrito é positivamente relacionado. Nesse caso, mesmo na presença de moderação da autoeficácia no fenômeno, as relações entre tipo de perfeccionismo e procrastinação permaneceram as mesmas (Bong et al., 2014).

\subsubsection{Aprendizagem autorregulada}

A aprendizagem autorregulada é o tipo de aprendizagem que envolve o controle dos processos cognitivos, das emoções e do comportamento. Estudantes autorregulados apresentam capacidade de escolha, bom processamento de informação, tomada de decisão, planejamento e responsabilidade pelas próprias ações (Boruchovitch, 2014). Em estudos que abordam as relações entre aprendizagem, procrastinação e autorregulação, a procrastinação é vista como positivamente associada com uma autorregulação deficiente, além de dificuldades atribuídas à falta de tempo. Ela também está negativamente associada com a percepção de uma boa preparação para a tarefa (González-Brignardello e Sánchez-Elvira-Paniagua, 2013).

Processos autorregulatórios também podem ser diferenciados pelo ambiente da aprendizagem e os objetivos do estudante ao ingressar num curso. Assim, por exemplo, 
em ambientes de aprendizagem online, estudantes de pós-graduação desenvolvem mais autorregulação e, portanto, procrastinam menos do que estudantes de graduação no mesmo ambiente (Artino e Stephens, 2009). De fato, processos de autorregulação parecem ser essenciais el ambiente de aprendizagem online. Cursos a distância que apresentam suporte de instrutores presenciais desenvolvem mais estudantes procrastinadores do que cursos totalmente a distância (Romano et al., 2005).

A aprendizagem autorregulada junto com outras variáveis são boas preditoras da procrastinação. Uma dessas variáveis -flow- é descrita como um estado de total envolvimento em uma atividade que consome a atenção completa do indivíduo (Csikszentmihaly, 1990). A procrastinação, por vezes, não é uma boa preditora do desempenho acadêmico, mas flow e aprendizagem autorregulada, quando trabalhados em conjunto, induzem ao bom desempenho acadêmico. Isso explica porque alguns estudos de procrastinação ativa (quando o indivíduo deliberadamente decide procrastinar) mostram correlação com bom resultados de aprendizagem. Provavelmente, alguns desses indivíduos regulam sua aprendizagem junto com estado de flow (Kim e Seo, 2013).

Outros conceitos têm sido ligados à aprendizagem autorregulada e procrastinação. Resolução (grit, no original) é considerada um traço de personalidade da pessoa que leva à perseverança e paixão por objetivos a longo prazo. Resolução tem sido ligada a indicadores da aprendizagem autorregulada. Em relação à procrastinação, indivíduos mais resolutos são menos propensos a procrastinar. Evidências apontam que resolução é associada com níveis reduzidos de atrasos para iniciar e completar tarefas acadêmicas (Wolters e Hussain, 2015). Ocorre que resolução é um fator protecional inibidor de comportamentos que atrapalham o desempenho, como pode ser o caso da procrastinação.

A perspectiva da aprendizagem autorregulada pode ser utilizada também para entender como a procrastinação está relacionada com a motivação. Para isso, é abordado o sistema de crenças metacognitivas do indivíduo. Crenças metacognitivas referem-se às informações que os indivíduos capturam sobre seus próprios estados cognitivos e internos, bem como sobre as estratégias adotadas em situações problemáticas (Cao, 2012). Indivíduos que procrastinam e utilizam a autorregulação metacognitiva apresentam níveis mais altos de ansiedade (Vahedi et al., 2012).

Ocorre que crenças sobre procrastinação são melhores preditores da procrastinação do que crenças sobre autoeficácia. Portanto, um indivíduo que acredita ser procrastinador (mas capaz de atingir os objetivos) tem maior tendência a procrastinar do que aquele que acredita não ser capaz de atingir o objetivo. A procrastinação ainda ocorre mais naquelas pessoas que acreditam que a procrastinação é benéfica (Cao, 2012).

A personalidade também possui muita influência na procrastinação mesmo quando há diferentes estratégias de autorregulação adotadas. Indivíduos com alta escrupulosidade tendem a procrastinar menos e usam um maior repertório de estratégias de autorregulação (Lubbers et al., 2010). Isso corrobora a ideia das relações estreitas entre escrupulosidade e procrastinação já adotadas pela literatura (Watson, 2001).

\subsubsection{Emoções e valores}

Emoções em ambientes de aprendizagem têm sido negligenciadas devido à crença popular da emoção em oposição ao pensamento racional, sendo este último o requisito necessário para a aprendizagem. Esse pensamento pode impedir a aprendizagem efetiva, 
além de desconsiderar o papel das emoções na experiência da aprendizagem como no seu desenvolvimento (You e Kang, 2014).

Exaustão, estresse, ansiedade e falta de interesse estão relacionados com procrastinação no ambiente acadêmico, especialmente em atividades típicas da área, como a escrita acadêmica (Lonka et al., 2014). Outros estudos relacionam ansiedade e procrastinação de forma direta (Macher et al., 2012). Não ter uma atitude positiva e não encarar uma tarefa como oportunidade também está relacionado com procrastinação (Peled e Sarid, 2010).

Estudantes com deficiência de aprendizagem possuem mais baixa inteligência emocional, baixa autoeficácia e índices mais altos de procrastinação. O efeito indireto da inteligência emocional na procrastinação é maior em estudantes com deficiência de aprendizagem. Portanto, estudantes que não procrastinam possuem maior habilidade em regularem seus estados emocionais (Hen e Goroshit, 2012).

Os valores sociais dos estudantes também estão relacionados com o comportamento procrastinatório. Os valores sociais são construídos pela necessidade humana de pertencimento (belongingness, no original) em relação ao contexto social. Os valores sociais podem ser avaliados de acordo com as preocupações do indivíduo em sua possível exclusão social. Nesse sentido, existe uma tendência a procrastinar naqueles indivíduos com baixo índice de pertencimento social. A procrastinação não foi associada aos valores associados às tarefas acadêmicas, ou seja, procrastinação pode ser mediador parcial da influência dos valores sociais no sentimento de pertencimento (Kennedy e Tuckman, 2013).

Valores culturais também têm influência na procrastinação acadêmica. As sociedades passaram de valores culturais modernos para pós-modernos. Estes evidenciam tolerância, apreciação de contatos sociais e autorrealização, já aqueles estão baseados em trabalho intenso, segurança e prosperidade. $\mathrm{O}$ estudante ainda voltado para valores modernos possui objetivos claros, esforça-se em completar tarefas desconfortáveis e deseja alcançar algo na vida. Os estudantes que desenvolveram valores culturais pósmodernos passam bastante tempo com amigos, apreciam diversão e atividades espontâneas e almejam uma vida de diversão. $O$ desenvolvimento de valores pósmodernos está relacionado com a procrastinação acadêmica (Dietz, Hofer e Fries, 2007).

Dentro de uma perspectiva social cognitiva, a procrastinação ajuda a entender também questões de suporte social no ambiente de aprendizagem. Estudantes que não procrastinam costumam procurar mais ajuda quando encontram dificuldades acadêmicas, já estudantes procrastinadores evitam ajuda em tarefas dificultosas (Zusho e Barnett, 2011). Adicionalmente, homens procrastinam mais do que mulheres pois procuram menos ajuda (Rodarte-Luna e Sherry, 2008).

Por fim, em projetos de longo prazo, como projetos de qualificação de tese, alguns indivíduos não possuem uma boa percepção de seus problemas de autocontrole e tornamse impacientes quando há uma maior proximidade do prazo final. Essas pessoas tendem a procrastinar, começam projetos que nunca terminam e podem finalizar projetos com qualidade inferior à esperada. Nesse caso, um sistema de bônus motivacional pode surtir efeito na procrastinação (Akin, 2012). 


\subsubsection{Ferramentas para redução}

Os estudos sobre ferramentas para redução da procrastinação em ambientes de aprendizagem estão basicamente associados às novas tecnologias de informação e comunicação, como a participação em cursos a distância mediados por computador. O trabalho cooperativo e a avaliação por pares, característicos desse tipo de aplicação, podem induzir a melhores desempenhos e resultados cognitivos nesses ambientes (Sampaio-Maia et al., 2013). Assim, partindo dessa premissa, alguns artigos selecionados tratavam de ferramentas ou estratégias para reduzir a procrastinação nesses ambientes e, com isso, promover a aprendizagem.

Pouca pesquisa empírica é realizada com o intuito de prevenir ou reduzir o comportamento procrastinatório. Para isso, abordagens experimentais podem ser utilizadas para investigar o processo de redução da procrastinação. Por exemplo, a utilização de uma ferramenta web de planejamento e protocolos de reflexão para sugerir aos estudantes uma monitoração do seu comportamento procrastinatório pode ser efetiva (Wäschle et al., 2014b). Nesse caso, o feedback visual foi fornecido por meio de um gráfico que mostrava ao estudante a evolução no curso e sua procrastinação prévia autorrelatada. A elaboração dessa ferramenta simples induziu os estudantes a reduzirem o seu comportamento procrastinatório.

Outros tipos de ferramentas foram construídos em estudos que promoviam a redução da procrastinação em ambientes de aprendizagem online (Alias, 2012). Foi construída uma ferramenta web com o objetivo de dar suporte às tarefas dos estudantes no ambiente online. A ferramenta possuía três funcionalidades: negociação de tarefas, status de acumulação de pontos e lembrete de tarefas. A ênfase estava no problema de sustentar a motivação dos estudantes ao utilizar um ambiente de e-learning. $\mathrm{O}$ suporte à motivação por meio da utilização da ferramenta foi efetivo na redução da procrastinação.

A questão da participação de estudantes nas ferramentas de aprendizado online também é discutida. Há indícios da conexão entre volição e sucesso no curso em ambientes assíncronos quando se quantifica o comportamento do estudante proativo em message boards (quadros de mensagens online) e se correlaciona esse comportamento com o sucesso. Ocorre que estudantes que postam mensagens mais cedo nos message boards do curso ganham notas que foram melhores do que a média final da turma, denotando que aqueles que procrastinaram a postagem de mensagem tiveram desempenho inferior (Warnock et al., 2012).

De fato, parece que procrastinadores são menos bem-sucedidos que nãoprocrastinadores, devido à sua falta de participação online. Foi encontrada uma relação negativa entre procrastinação e desempenho acadêmico e essa relação foi mediada pelo nível de participação nos fóruns de discussão online. Os alunos que procrastinam mais a participação em discussões online utilizando as ferramentas web apresentam mais incidências de recomeço do curso a distância, sentem-se menos motivados para trabalhar no curso e, usualmente, deixam de frequentar o curso (Michinov et al., 2011).

Por fim, para auxiliar na apreensão das proposições apresentadas nesta seção foi elaborada a figura 2, onde é apresentado um diagrama esquemático das principais conexões entre os conceitos resultantes da RSL. 


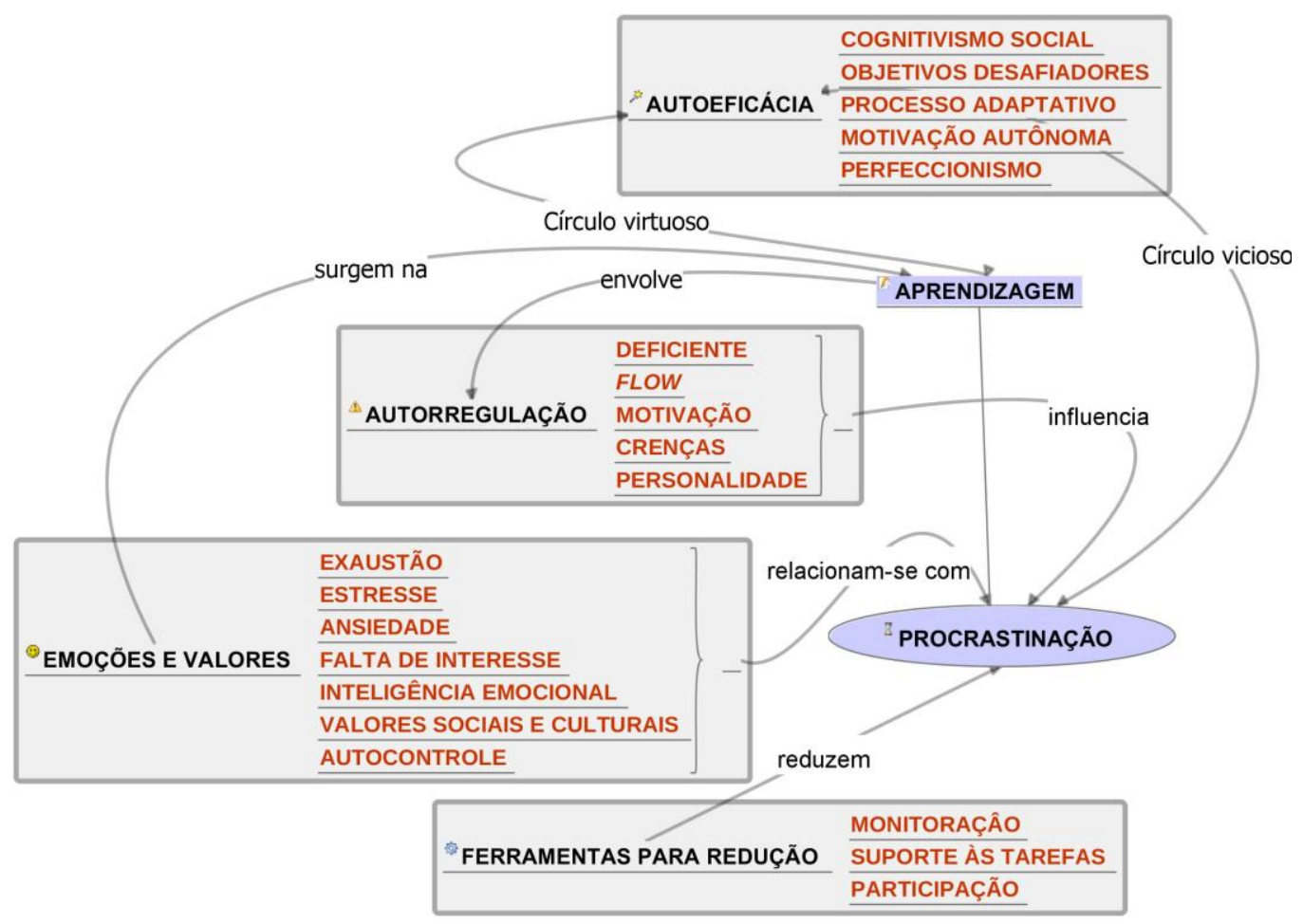

Figura 2. Mapa conceitual entre aprendizagem e procrastinação Fonte: Elaborado pelos autores no software.

\section{Conclusões}

Não é a esmo que os estudos sobre procrastinação focam primordialmente em tarefas relacionadas com o ambiente acadêmico e aprendizagem. Ao ser encarada como um comportamento de fracasso autorregulado, a procrastinação é um reflexo de crenças, elaboração de estratégias e emoções que permeiam o âmbito da aprendizagem. A revisão sistemática da literatura aqui apresentada deu suporte a essa proposição, além de reafirmar as relações entre os dois fenômenos. O objetivo foi reunir as evidências científicas dos últimos dez anos sobre as relações conceituais entre procrastinação e aprendizagem. Tais evidências falam principalmente das associações de autoeficácia, autorregulação, emoções e valores com a procrastinação, todas envolvendo o ambiente da aprendizagem. Também foram encontradas proposições de algumas ferramentas para reduzir a procrastinação nesse ambiente. A RSL elaborada permitiu categorizar e discutir os principais conceitos envolvendo procrastinação e aprendizagem. Dessa maneira, a principal contribuição deste trabalho é destacar esses conceitos que permeiam os dois fenômenos. Com esse resultado, é possível seguir passos adicionais em estudos posteriores.

Logo, estudos futuros poderão utilizar os conceitos reunidos como base para a investigação empírica. Ainda não é explícita a influência da procrastinação em processos de aprendizagem. As evidências que autoeficácia, autorregulação, emoções e valores influenciam os dois fenômenos podem abrir caminho para a construção de uma rede nomológica de construtos e desenvolvimento de teoria. Para isso, estudos futuros 
deverão investigar se a relação entre procrastinação e aprendizagem é direta ou se os conceitos apontados mediam essa relação.

Também foram encontrados estudos que tratavam do papel de ferramentas tecnológicas na redução da procrastinação no ambiente de aprendizagem, por meio de monitoração, suporte às tarefas e participação. Esses resultados podem servir a estudos futuros que tratem de abordagens experimentais com o propósito de reduzir a procrastinação em ambientes de aprendizagem. Assim, deverão ser exploradas outras possibilidades permitidas pelo desenvolvimento da tecnologia, de modo a evitar o adiamento de atividades.

Fortuitamente ou não, os estudos selecionados possuem um viés para uma visão epistemológica objetiva da realidade e uma perspectiva social cognitiva do fenômeno da aprendizagem. De certa forma, isso limita a visão das relações entre procrastinação e aprendizagem. Em sua maioria, os estudos não são capazes de explicar resultados conflitantes quando "se deixa para fazer amanhã o que era para fazer hoje". Sugere-se que estudos futuros sobre procrastinação possam explorar novas abordagens da aprendizagem, como a abordagem construtivista que cria sentido a partir da experiência.

Ademais, a exploração de abordagens qualitativas, ou seja, onde há mais proximidade do pesquisador com o fenômeno é importante para ampliar seu entendimento. Ainda que uma revisão sistemática apresente um rigor metodológico, ela não esgota o conhecimento disponível. Portanto, a principal limitação deste trabalho deriva da seleção das fontes para coleta dos estudos primários. Embora a base selecionada possua ampla cobertura da publicação científica mundial, algum conceito relacionando procrastinação e aprendizagem pode não ter sido abordado. Nesse caso, entende-se que o investimento em abordagens empíricas nas quais é possível a emergência de conceitos pode minimizar esse efeito. $\mathrm{O}$ foco passaria para algum conceito não explorado nesta revisão.

Como implicações para a prática, profissionais de diversas áreas podem focar nos conceitos especificados como relacionados entre procrastinação e aprendizagem para tomada de decisão em ambientes pedagógicos ou acadêmicos. Processos autorregulatórios e de autoeficácia devem ser monitorados por gestores acadêmicos ou educadores, por exemplo, para que não venham a interferir em prazos na entrega de trabalhos ou projetos. Aprendizes devem estar atentos às emoções surgidas durante o processo de aprendizagem para avaliar sua interferência no comportamento procrastinador.

\section{Referências}

Akin, Z. (2012). Intertemporal decision making with present biased preferences. Journal of Economic Psychology, 33(1), 30-47. http://doi.org/10.1016/j.joep.2011.09.007

Alias, N. A. (2012). Design of a motivational scaffold for the Malaysian e-learning environment. Educational Technology \& Society, 15(1), 137-151.

Artino, A. R. e Stephens, J. M. (2009). Academic motivation and self-regulation: A comparative analysis of undergraduate and graduate students learning online. Internet and Higher Education, 12(3-4), 146-151. doi:10.1016/j.iheduc.2009.02.001

Bandura, A. (1977). Self-efficacy: Toward a unifying theory of behavioral change. Psychological Review, 84(2), 191-215. doi:10.1037/0033-295X.84.2.191 
Blair, J. (2008). Resistance is utile. English Studies in Canada, 34(2-3), 11-15. doi:10.1353/esc.0.0122

Bong, M., Hwang, A., Noh, A. e Kim, S. (2014). Perfectionism and motivation of adolescents in academic contexts. Journal of Educational Psychology, 106(3), 711-729. doi: $10.1037 / \mathrm{a} 0035836$

Boruchovitch, E. (2014). Autorregulação da aprendizagem: Contribuições da psicologia educacional para a formação de professores. Psicologia Escolar e Educacional, 18(3), 401409. doi:10.1590/2175-3539/2014/0183759

Bruhm, S. (2008). What I didn't do on my summer vacation. English Studies in Canada, 34(2-3), 21-24. doi:10.1353/esc.0.0136

Cao, L. (2012). Differences in procrastination and motivation between undergraduate and graduate students. Journal of the Scholarship of Teaching and Learning, 12(2), 39-64.

Carnell, E. e Lodge, C. (2001). Supporting effective learning. Londres: SAGE Publications Ltd.

Case, J. (2009). Resource restricted computability theoretic learning: Illustrative topics and problems. Theory of Computing Systems, 45(4), 773-786. doi:10.1007/s00224-009-9169-7

Chu, A. H. C. e Choi, J. N. (2005). Rethinking procrastination: Positive effects of "active" procrastination behavior on attitudes and performance. The Journal of Social Psychology, 145(3), 245-264. doi:10.3200/SOCP.145.3.245-264

Corkin, D. M., Yu, S. L. e Lindt, S. F. (2011). Comparing active delay and procrastination from a self-regulated learning perspective. Learning and Individual Differences, 21(5), 602-606. doi:10.1016/j.lindif.2011.07.005

Csikszentmihaly, M. (1990). Flow: The psychology of optimal experience. Nueva York: Harper \& Row.

De Moura, P. J. e Bellini, C. G. P. (2013). Antecedentes e consequentes de flow em equipes de trabalho: Revisão da literatura e aplicações em tecnologia da informação. Revista Brasileira de Administração Científica, 4(2), 6-23. doi:10.6008\%2FESS2 179-684X.2013.002.0004

Dietz, F., Hofer, M. e Fries, S. (2007). Individual values, learning routines and academic procrastination. British Journal of Educational Psychology, 77(4), 893-906. doi:10.1348/000709906X169076

Finigan, T. (2008). Expect delays: Procrastination and the graduate student. English Studies in Canada, 34(2-3), 1-7. doi:10.1353/esc.0.0141

González-Brignardello, M. P. e Sánchez-Elvira-Paniagua, Á. (2013). ¿Puede amortiguar el engagement los efectos nocivos de la procrastinación académica? Acción Psicológica, 1O(1), 115-134. doi:10.5944/ap.10.1.7039

Haghbin, M., McCaffrey, A. e Pychyl, T. A. (2012). The complexity of the relation between fear of failure and procrastination. Journal of Rational-Emotive \& Cognitive-Behavior Therapy, 30(4), 249-263. doi:10.1007/s10942-012-0153-9

Harris, N. e Sutton, R. (1983). Task procrastination in organizations: A framework for research. Human Relations, 36(11), 987-996. doi:10.1177/001872678303601102

Hederih, B. R. e Čagran, B. (2014). Učno zavlačevanje pri srednješolcih. Revija za Elementarno Izobraževanje, 2, 117-128.

Hen, M. e Goroshit, M. (2012). Academic procrastination, emotional intelligence, academic selfefficacy, and GPA: A comparison between students with and without learning disabilities. Journal of Learning Disabilities, 47(2), 1-24. doi:10.1177/0022219412439325 
Howell, A. e Watson, D. (2007). Procrastination: Associations with achievement goal orientation and learning strategies. Personality and Individual Differences, 43, 167-178. doi:10.1016/j.paid.2006.11.017

Katz, I., Eilot, K. e Nevo, N. (2014). I'll do it later: Type of motivation, self-efficacy and homework procrastination. Motivation and Emotion, 38(1), 111-119. doi:10.1007/s11031013-9366-1

Kennedy, G. J. e Tuckman, B. (2013). An exploration into the influence of academic and social values, procrastination, and perceived school belongingness on academic performance. Social Psychology of Education, 16(3), 435-470. doi:10.1007/s11218-013-9220-Z

Kim, C. (2012). The role of affective and motivational factors in designing personalized learning environments. Educational Technology Research and Development, 60(4), 563-584. http://doi.org/10.1007/s1 1423-012-9253-6

Kim, E. e Seo, E. H. (2013). The relationship of flow and self-regulated learning to active procrastination. Social Behavior and Personality, 41(7), 1099-114. doi:10.2224/sbp.2013.41.7.1099

Kitchenham, B., Brereton, O. P., Budgen, D., Turner, M., Bailey, J. e Linkman, S. (2009). Systematic literature reviews in software engineering. A systematic literature review. Information and Software Technology, 51(1), 7-15. doi:10.1016/j.infsof.2008.09.009

Klingsieck, K. B., Fries, S., Horz, C. e Hofer, M. (2012). Procrastination in a distance university setting. Distance Education, 33, 37-41. doi:10.1080/01587919.2012.723165

Knowles, M. S., Holton, E. F. e Swanson, R. A. (2011). The adult learner: The definitive classic in adult education and human resource management. Burlington: Elsevier.

Lay, C. H. (1986). At last, my research article on procrastination. Journal of Research in Personality, 20(4), 474-495. doi: 10.1016/0092-6566(86)90127-3

Lonka, K., Chow, A., Keskinen, J., Hakkarainen, K., Sandström, N. e Pyhältö, K. (2014). How to measure PhD. students' conceptions of academic writing, and are they related to wellbeing? Journal of Writing Research, 5(3), 245-269. doi:10.17239/jowr-2014.05.03.1

Lubbers, M. J., Van der Werf, M. P. C., Kuyper, H. e Hendriks, A. A. J. (2010). Does homework behavior mediate the relation between personality and academic performance? Learning and Individual Differences, 20(3), 203-208. doi:10.1016/j.lindif.2010.01.005

Macher, D., Paechter, M., Papousek, I. e Ruggerii, K. (2012). Statistics anxiety, trait anxiety, learning behavior, and academic performance. European Journal of Psychology of Education, 27(4), 483-498. doi:10.1007/s10212-011-0090-5

Merriam, S. B. e Bierema, L. L. (2014). Adult learning: Linking theory and practice. San Francisco, CA: Jossey-Bass.

Michinov, N., Brunot, S., Le Bohec, O., Juhel, J. e Delaval, M. (2011). Procrastination, participation, and performance in online learning environments. Computers and Education, 56(1), 243-252. doi:10.1016/j.compedu.2010.07.025

O’Driscoll, M. (2008). Getting around to it: Revaluing procrastination. English Studies in Canada, 34(2-3), 1-3. doi:10.1353/esc.0.0118

Ozer, B. U., Demir, A. e Ferrari, J. R. (2013). Reducing academic procrastination through a group treatment program: A pilot study. Journal of Rational-Emotive \& Cognitive-Behavior Therapy, 31(3), 127-135. doi:10.1007/s10942-013-0165-0 
Panadero, E. e Alonso-Tapia, J. (2014). How do students self-regulate? Review of Zimmerman's cyclical model of self-regulated learning. Anales de Psicología, 30(2), 450-462. doi:10.6018/analesps.30.2.167221

Peled, Y. e Sarid, M. (2010). Multi-drafting feedback process in a web-based environment. Interactive Technology and Smart Education, 7(2), $113-123$. doi:10.1108/17415651011071046

Pychyl, T. A. (2008). Savouring the flavours of delay. English Studies in Canada, 34(2-3), 25-29. doi:10.1353/esc.0.0113

Raelin, J. A. (2009). Action learning and related modalities. En S. J. Armstrong e C. V. Fukami, The SAGE handbook of management learning, education and development (pp. 419-438). Londres: Sage.

Rodarte-Luna, B. e Sherry, A. (2008). Sex differences in the relation between statistics anxiety and cognitive/learning strategies. Contemporary Educational Psychology, 33(2), 327-344. doi: 10.1016/j.cedpsych.2007.03.002

Romano, J., Wallace, T. L., Helmick, I. J., Carey, L. M. e Adkins, L. (2005). Study procrastination, achievement, and academic motivation in web-based and blended distance learning. Internet and Higher Education, 8(4), 299-305. doi:10.1016/j.iheduc.2005.09.003

Sampaio, R. F. e Mancini, M. C. (2007). Estudos de revisão sistemática: Um guia para síntese criteriosa da evidência científica. Revista Brasileira de Fisioterapia, 11(1), 83-89. doi:10.1590/S1413-35552007000100013

Sampaio-Maia, B., Maia, J. S., Leitão, S., Amaral, M. e Vieira-Marques, P. (2013). Wiki as a tool for microbiology teaching, learning and assessment. European Journal of Dental Education, 18(2), 91-97. doi:10.1111/eje.12061

Seo, E. H. (2013). A comparison of active and passive procrastination in relation to academic motivation. Social Behavior and Personality, 41(5), 777-786. doi:10.2224/sbp.2013.41.5.777

Sugiyama, M., Hirowatari, E., Tsuiki, H. e Yamamoto, A. (2013). Learning figures with the Hausdorff metric by fractals. Towards computable binary classification. Machine Learning, 9O(1), 91-126. doi:10.1007/s10994-012-5301-Z

Tice, D. e Baumeister, R. (1997). Longitudinal study of procrastination, performance, stress, and health: The cost and benefits of dawdling. Psychological Science, 8(6), 454-458. doi:10.1111/j.1467-9280.1997.tb00460.x

Vahedi, S., Farrokhi, F., Gahramani, F. e Issazadegan, A. (2012). The relationship between procrastination, learning strategies and statistics anxiety among Iranian college students: A canonical correlation analysis. Iranian Journal of Psychiatry and Behavioral Sciences, 6(1), $40-46$.

Van Eerde, W. (2003). A meta-analytically derived nomological network of procrastination. Personality and Individual Differences, 35(6), 1401-1418. doi:10.1016/So191-8869(02)003586

Warnock, S., Bingham, K., Driscoll, D., Fromal, J. e Rouse, N. (2012). Early participation in asynchronous writing environments and course success. Journal of Asynchronous Learning Networks, 16(1), 35-47.

Wäschle, K., Allgaier, A., Lachner, A., Fink, S. e Nückles, M. (2014a). Procrastination and selfefficacy: Tracing vicious and virtuous circles in self-regulated learning. Learning and Instruction, 29, 103-114. doi:10.1016/j.learninstruc.2013.09.005 
Wäschle, K., Lachner, A., Stucke, B., Rey, S., Frömmel, C. e Nückles, M. (2014b). Effects of visual feedback on medical students' procrastination within web-based planning and reflection protocols. Computers in Human Behavior, 41, 120-136. doi:10.1016/j.chb.2014.09.022

Watson, D. (2001). Procrastination and the five-factor model: A facet level analysis. Personality and Individual Differences, 30, 149-158. doi:10.1016/So191-8869(00)00019-2

Wolters, C. A. e Benzon, M. B. (2013). Assessing and predicting college students' use of strategies for the self-regulation of motivation. Journal of Experimental Education, 81(2), 199-22 1. doi:10.1080/00220973.2012.699901

Wolters, C. A. e Hussain, M. (2015). Investigating grit and its relations with college students' self-regulated learning and academic achievement. Metacognition and Learning, 10(3), 293311. doi:10.1007/s1 1409-014-9128-9

Wright, J. M. (2008). Pathologizing procrastination; or, the romanticization of work. English Studies in Canada, 34(2-3), 16-20. doi:10.1353/esc.0.0135

Yeo, G. e Neal, A. (2013). Revisiting the functional properties of self-efficacy: A dynamic perspective. Journal of Management, 39(6), 1385-1396. doi:10.1177/0149206313490027

You, J. W. e Kang, M. (2014). The role of academic emotions in the relationship between perceived academic control and self-regulated learning in online learning. Computers and Education, 77, 125-133. doi:10.1016/j.compedu.2014.04.018

Zusho, A. e Barnett, P. A. (2011). Personal and contextual determinants of ethnically diverse female high school students' patterns of academic help seeking and help avoidance in English and mathematics. Contemporary Educational Psychology, 36(2), 152-164. doi:10.1016/j.cedpsych.2011.02.002

\section{Breve CV de los autores}

\section{Rony Rodrigues Correia}

Doutorando em Administração, Mestre em Administração e Bacharel em Engenharia de Produção, todos pela Universidade Federal da Paraíba. Especialista em Desenvolvimento de Sistemas Web pela União dos Institutos Brasileiros de Tecnologia. Revisor do periódico Journal of Internet Banking and Commerce e avaliador de eventos nacionais brasileiros como EnANPAD e SemeAd. Larga experiência profissional na área de Tecnologia da Informação, trabalhando nessa área na Universidade Federal da Paraíba. Autor de publicações em periódicos e congressos nacionais. Pesquisador assistente do GTIS - Grupo de Pesquisa em Tecnologia da Informação e Sociedade, certificado pelo Conselho Nacional de Desenvolvimento Científico e Tecnológico (CNPq). ORCID ID: 0000-0002-1324-6494. Email: ronyrc@gmail.com

\section{Pedro Jácome de Moura Júnior}

Professor do Departamento de Administração e Superintendente de Tecnologia da Informação da Universidade Federal da Paraíba. Doutor e Mestre em Administração também pela Universidade Federal da Paraíba. Líder do ODIN - Observatório de Dados Institucionais e pesquisador no GTIS - Grupo de Pesquisa em Tecnologia da Informação e Sociedade, ambos certificados pelo Conselho Nacional de Desenvolvimento Científico e Tecnológico (CNPq). Autor de publicações em periódicos nacionais e internacionais como Computers in Human Behavior (CHB), Journal of Global IT 
R. Correia y P. J. de Moura

Management (JGITM), Journal of Information Systems and Technology Management (JISTEM) e Cadernos EBAPE. Possui trabalhos apresentados em conferências nacionais e internacionais. ORCID ID: 0000-0001-6548-4614. Email: pjacome@sti.ufpb.br 